Céline Bessière Frédérique Houseaux

\title{
Suivre des enquêteurs
}

In: Genèses, 29, 1997. pp. 100-114.

Citer ce document / Cite this document :

Bessière Céline, Houseaux Frédérique. Suivre des enquêteurs. In: Genèses, 29, 1997. pp. 100-114.

doi : 10.3406/genes.1997.1482

http://www.persee.fr/web/revues/home/prescript/article/genes_1155-3219_1997_num_29_1_1482 


\section{Suivre des enquêteurs}

\author{
Céline Bessière \\ Frédérique Houseaux
}

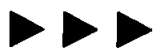

1. Les ouvriers agricoles, autrefois nombreux dans les régions de grande culture, constituaient alors un prolétariat rural particulièrement pauvre et démuni culturellement (voir Michel Gervais, Marcel Jollivet, Yves Tavernier, La Fin de la France paysanne de 1914 à nos jours, tome 4 de l'Histoire de la France rurale, Paris, Éd. du Seuil, 1976, pp. 253-256). La mécanisation de l'agriculture a supprimé la plus grande part de ces emplois très peu qualifiés de domestiques de ferme, recrutés dans certaines régions parmi les enfants de l'Assistance Publique (voir Anne Cadoret, Parenté plurielle. Anthropologie du placement familial, Paris, L'Harmattan, 1995). Les salariés agricoles qui ont subsisté malgré la mécanisation sont souvent devenus des mécaniciens agricoles relativement qualifiés, capables de conduire et de réparer leurs machines. Cependant, dans certaines régions, leur statut antérieur s'est perpétué, du fait en particulier de la dépendance personnelle qui les lie à leur patron, de leur célibat fréquent, de leur absence d'avenir. C'est, semble-t-il, le cas de Serge. et article est issu d'une expérience de
terrain un peu particulière. Lors d'une
passation de questionnaire à laquelle nous assistions en observatrices, le questionnaire, à l'élaboration duquel nous avions participé, nous est apparu particulièrement inadapté à la personne interrogée, un salarié agricole. Cette confrontation du questionnaire au terrain aurait pu nous mener à dresser un constat d'échec et à rallier le camp des détracteurs de la statistique: appliquer un questionnaire standardisé à des personnes fondamentalement différentes ne peut prétendre décrire correctement la réalité telle que ces personnes la vivent.

Mais, dans la mesure où nous avions pris de nombreuses précautions pour garantir la fiabilité et la pertinence du questionnaire (entretiens préparatoires, questions directement issues de ces entretiens, leçons tirées d'enquêtes antérieures...), il nous a semblé préférable d'assumer ces observations dans l'exploitation et l'interprétation des données, et de s'en servir comme base pour une réflexion d'ordre méthodologique: en effet, au-delà des failles du questionnaire, cette expérience a surtout révélé l'importance de la situation d'enquête en sociologie quantitative.

\section{L'ouvrier, sa patronne et l'enquêtrice}

L'enquêté, que nous appellerons Serge, est ouvrier agricole ${ }^{1}$. Il est interrogé sur son lieu de travail, en présence de sa patronne qui le prend ouvertement pour un incapable (incapable en particulier de répondre à une enquête), le terrorise et répond à sa place, ce qui conduit finalement l'enquêtrice à prendre un autre rendez-vous avec Serge, hors de la présence de sa patronne.

L'enquêtrice est accueillie par l'exploitante agricole, patronne de Serge. Celle-ci tente immédiatement de la dissuader de faire 


\section{Une enquête sur l'enquête: le séminaire Bonheur et travail}

Le dispositif d'enquêtes permanentes sur les Conditions de Vie de l'Insee correspond chaque année à trois enquêtes distinctes en janvier, mai et octobre ( 8000 ménages contactés, près de 6000 observations). Chacune de ces enquêtes comprend elle-même deux parties. La première, dite "indicateurs sociaux», est destinée à appréhender et à mesurer régulièrement certains aspects de la vie des ménages. La seconde, dite «variable», est destinée à appréhender ponctuellement tel ou tel problème social selon les priorités du moment.

L'enquête de janvier 1997 traitait en première partie des questions de qualité de l'habitat et de l'environnement. La seconde partie, intitulée «Travail et modes de vie», cherchait à explorer la place que les différents individus attribuent aujourd'hui au travail dans leur existence ( $y$ compris lorsqu'ils se trouvent inactifs, au chômage ou occupent des emplois précaires) et à identifier les différents aspects du travail qui peuvent les rendre heureux ou malheureux. Composée d'une soixantaine de questions, dont 6 questions ouvertes, elle était censée durer 30 minutes environ pour une première partie un peu plus longue.

Le questionnaire «Travail et modes de vie» a été conçu dans le cadre du séminaire Bonheur et travail de l'École Normale Supérieure. Composé d'une quinzaine de participants, de formations et d'origines différentes (sociologues, philosophes, économistes, historiens), de jeunes en formation et de professionnels plus chevronnés réunis autour de Christian Baudelot, il a bénéficié de l'expérience de Michel Gollac, qui a beaucoup utilisé en statisticien les enquêtes sur les conditions de travail, de celle de Stéphane Beaud, familier de l'analyse ethnographique du travail et du chômage dans la région de Montbéliard, de celle d'Anne Flipo, statisticienne de l'Insee connaissant très bien les procédures d'enquêtes. Les premiers résultats de l'enquête ont été publiés dans C. Baudelot, M. Gollac, «Faut-il travailler pour être heureux?» Insee Première, $n^{\circ} 560$, déc. 1997.

Les questions ont été élaborées à partir de vingt-cinq entretiens discutés, comparés et analysés par les participants au séminaire et réalisés auprès de personnes dont les conditions de travail et d'emploi étaient les plus contrastées possible. Le questionnaire, dans ses différentes versions préparatoires, a été testé deux fois dans quatre régions différentes avant la passation finale.

En tant qu'étudiantes participant au séminaire, nous avons eu l'occasion de suivre des enquêteurs de l'Insee pendant la période de passation. Ce sont les observations que l'ensemble des participants ont recueillies auprès des enquêteurs au cours de cette «enquête sur l'enquête» qui servent de support aux quelques réflexions présentées ici.

l'enquête: «Il ne pourra jamais vous répondre, il en est incapable... Donnez-moi un exemple des questions que vous voulez lui poser... C'est beaucoup trop philosophique pour lui! Il vous dira seulement oui, non... Un exemple: il sait à peine écrire, même pas recopier une lettre! En plus il passe son temps au bistrot!». En outre, il est clair que sa patronne envisage sans plaisir un entretien qui, prenant sur le temps de travail de Serge, bouleverserait le chantier de la matinée. L'enquêtrice négocie alors une nouvelle visite pour l'heure du déjeuner.

Lorsqu'elle revient, vers 13 heures, l'exploitante agricole insiste pour que la passation du questionnaire se fasse assis à la table de la salle à manger, c'est-à-dire au milieu de la pièce parmi ses deux enfants et elle-même. L'enquêtrice demande si elle ne peut pas s'installer dans un coin du salon, à l'écart, mais l'agricultrice refuse expressément: "Vous serez mieux sur la table et je ne voudrais pas que Serge salisse les fauteuils». C'est alors que Serge entre dans la pièce, appelé par sa patronne: c'est un homme de 35 ans, en bleu de travail. Bien que sa patronne lui demande de s'asseoir, il n'ose pas, reste en retrait, dans le dos de l'enquêtrice, debout, totalement paralysé par la situation.

L'enquêtrice commence à dérouler le questionnaire à voix basse car la patronne, bien que vaquant à ses occupations autour de la table, surveille le déroulement de l'enquête. L'enquêtrice omet ainsi toute la première page du questionnaire, notamment la question «Qu'est-ce qui pour vous est le plus important pour être heureux?», elle avouera ensuite ne pas avoir osé la poser. 


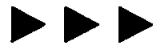

2. Voir entre autres les textes classiques de Basil Bernstein, Langage et classes sociales. Codes socio-linguistiques et contrôle social, présentation par Jean-Claude Chamboredon, Paris, Minuit, 1975, et de William Labov, Le parler ordinaire. La langue dans les ghettos noirs des Etats-Unis, Paris, Minuit 1978. Ces questions ont été plus souvent abordées dans le cadre des discussions sur l'entretien (voir Liliane Kandel, «Réflexions sur l'usage de l'entretien, notamment non directif, et sur les études d'opinions», Epistémologie sociologique, $n^{\circ} 13,1^{\text {er }}$ semestre 1972) que de celles sur le questionnaire. On peut aussi se reporter à Pierre Bourdieu, "Questions de politique », Actes de la recherche en sciences sociales, $n^{\circ} 16$, sept. 1977 , pp. 76-82. Une excellente synthèse des discussions autour de l'entretien et du questionnaire est effectuée, à partir de la discussion d'une enquête particulière, par Willy Pelletier, «Questionnaire et promotions de soi. Quelques hypothèses autour d'un merci», Critiques sociales, $\mathrm{n}^{\circ} 8-9$, juin 1996, pp.16-54.

3. Pierre Bourdieu, Jean-Claude Chamboredon, Jean-Claude Passeron, Le Métier de sociologue, Paris, Mouton/Bordas, 1968, p. 70.
Le dialogue est difficile. Serge ne comprend pas toutes les questions, ose à peine parler à haute voix. La patronne rôde de plus en plus autour de la table, dissimule mal un sourire devant la difficulté de son employé à répondre aux questions de l'Insee. Elle va jusqu'à répondre à sa place pour les questions concernant les horaires, le temps de travail. L'enquêtrice est alors exaspérée par la situation. Elle tourne furieusement les pages du questionnaire, pose une question de-ci de-là, parmi les plus descriptives, avant d'annoncer à Serge qu'elle prendra rendez-vous avec lui pour reprendre le questionnaire à son domicile.

Cet exemple cumule à lui seul la plupart des difficultés que nous avons recensées lors de ces passations et qui correspondent pour une part à des problèmes classiques de l'enquête par questionnaire liés au rapport au langage ${ }^{2}$, mais qui posent plus fondamentalement la question de la place faite à la situation d'enquête dans l'élaboration et l'exploitation du questionnaire, en particulier le rôle joué par l'enquêteur.

\section{Le sens des mots}

À la base de toute enquête statistique, un présupposé universaliste: les questions posées ont un sens pour chacun et si possible... le même sens. C'est au niveau des réponses que doivent s'exprimer les différences. L'intérêt irremplaçable d'une enquête statistique sur la totalité de la population est précisément de dresser la carte de cet ensemble de variations.

Mais surgit alors une contradiction. Plus la population est large et diverse, et plus l'enquête statistique s'impose car elle est la seule à pouvoir se faire une idée des grandes dimensions qui structurent le champ ainsi que du poids respectif de chacune des fractions qui constituent l'ensemble. Mais, inversement, plus la population est vaste et plus elle est diverse, plus il est difficile par conséquent de 
la soumettre à un questionnement qui ait le même sens pour tous.

«Supposer que la même question a le même sens pour des sujets sociaux séparés par les différences de culture associées aux appartenances de classe, c'est ignorer que les différents langages ne diffèrent pas seulement par l'étendue de leur lexique ou leur degré d'abstraction mais aussi par les thématiques et les problématiques qu'ils véhiculent ${ }^{3}$.»

\section{Questionnaire et compétence linguistique}

Dans une première approche, suivre des enquêteurs Insee, c'est prendre conscience de l'existence de niveaux différents de langage selon la position sociale de l'enquêté. C'est ce qui s'est passé avec Serge, pour qui la quasitotalité du questionnaire était incompréhensible du fait du décalage entre la lettre du questionnaire et la compétence linguistique de l'enquêté.

L'existence de ce jeu entre les niveaux de langage du questionnaire et ceux de l'enquêté conduit l'enquêteur à reformuler les questions dans un lexique qui lui semble adapté. Toute la difficulté consiste dans le fait qu'il n'y a aucune trace de cette intervention ni aucun moyen d'en juger la pertinence lors de l'exploitation des données: comment savoir dès lors à quelle question a répondu l'enquêté?

Ainsi, à une femme de ménage qui ne comprenait pas ce qu'on lui demandait par «Devez-vous travailler à un rythme élevé?» (« un rythme, vous voulez dire une ambiance?»), l'enquêtrice a demandé: «Est-ce que vous devez travailler vite? », reprise de la question plutôt neutre à nos yeux. Mais la reformulation pose plus de problèmes quand cette même personne, ne comprenant pas ce qu'était une promotion, a dâ se faire expliquer le terme par l'enquêtrice qui lui a répondu «c'est changer de travail». Or cette personne allait passer à temps plein, ce qui constituait pour elle un changement important dans son travail, mais l'enquêtrice, en dernier ressort, n'a pas voulu considérer ce changement comme une promotion. En reformulant d'une façon réductrice la question, l'enquêtrice s'est donc trouvée prisonnière d'un malentendu qui a rendu impossible toute réponse à la question initiale. Ce simple constat de différence des niveaux de langage nous permet déjà de percevoir l'importance du rôle de l'enquêteur comme médiateur entre le lexique du questionnaire et celui de l'enquêté, avec toutes les difficultés que comporte ce rôle; nous reviendrons sur ce statut de l'enquêteur dans la passation.

\section{Des sens dans tous les sens}

Au-delà des niveaux de langage, les enquêtés se différencient également par la réalité sociale que leur langage exprime et dont il est tributaire. Le sens que l'enquêté donne à une question peut être, de façon imprévue, un sens complètement différent de celui auquel les concepteurs du questionnaire avaient pensé.

Le meilleur exemple en est peut-être la question suivante: «Vous arrive-t-il d'éprouver dans votre travail l'impression que ce que vous faites, n'importe qui pourrait le faire?» Le libellé de cette question nous a été suggéré par un sociologue enquêtant parmi des chauffeurs d'autobus de la banlieue parisienne. S'exprimait dans cette formule, très répandue à la RATP, le sentiment assez dévalorisant d'accomplir un travail qui ne réclamait pas de qualifications rares (conduire) et qui condamnait ceux qui l'exerçaient à se considérer comme des pions remplaçables du jour au lendemain par n'importe qui.

Ce libellé permettait de condenser, de façon économique et concrète, un très grand nombre d'attitudes que nous avions rencontrées chez d'autres travailleurs et qui exprimaient, souvent douloureusement, le sentiment de leur interchangeabilité permanente. 
Elle avait aussi le mérite de pouvoir s'opposer aux attitudes inverses, plus fréquentes chez les salariés plus qualifiés ou diplômés, où la fierté que tire le travailleur de son travail provient de la rareté et de la complexité de sa qualification ou de la nature de son travail. C'est ce que signifiaient des formules comme «l'impression d'être reconnu à sa juste valeur», «imprimer une marque personnelle», «exercer une influence sur le cours des choses», «le plaisir de contempler les produits ou les résultats de mon travail», recueillies au cours des entretiens préliminaires à la fabrication du questionnaire.

Dans notre esprit, répondre «non » à la question «Vous arrive-t-il d'éprouver dans votre travail l'impression que ce que vous faites, n'importe qui pourrait le faire?» était donc le signe que l'enquêté se trouvait valorisé dans son travail. Au contraire, nous nous attendions à ce que les réponses «oui» soient le fait de personnes qui avaient l'impression d'être des «rouages anonymes», c'est-à-dire des personnes qui pensaient faire un travail tellement peu qualifié qu'elles pouvaient être remplacées par n'importe qui et n'importe quand. Or, au cours des passations, nous avons rencontré deux femmes de ménage qui nous ont répondu l'une «oui » et l'autre «non» dans un sens totalement différent de celui auquel nous nous attendions. Pour l'une, la réponse à cette question fut «Non, tout le monde ne pourrait pas faire ce que je fais, parce que c'est un travail tellement éprouvant que tout le monde ne le supporterait pas» c'està-dire que pour elle, la réponse était «non», mais l'origine de ce «non» était que son travail était dévalorisant. Pour elle, être irremplaçable était le signe de la déconsidération de sa tâche: personne ne voudrait faire ça telle-

4. J.-C. Passeron, «Le langage de l'abstention », in «Le questionnement et le silence», Consommation, $n^{\circ} 4,1982$.

5. P. Bourdieu, «L'opinion publique n'existe pas», Questions de sociologie, Paris, Minuit, 1984, p. 222. ment c'est bas, il n'y a que moi qui suis descendue assez bas pour accepter ça.

Pour l'autre femme de ménage au contraire, la réponse fut «Oui, c'est un travail normal, je 
suis comme une autre! », c'est-à-dire «oui» non pas parce que son travail aurait été perçu comme un travail dévalorisant mais au contraire parce qu'il était aussi honorable qu'un autre. Si n'importe qui peut faire ce travail, c'est que c'est un emploi «normal», c'està-dire gratifiant dans un milieu social où l'on est toujours guetté par la précarisation. Visiblement, ce sont aussi leurs conditions de travail qui conduisent ces deux femmes à donner un sens différent de celui des chauffeurs d'autobus à cette expression. Leurs conditions de travail difficiles les conduisent à trouver une identité valorisante là où d'autres, mieux servis, n'y verraient que dégradation de l'individu.

Ainsi, ce sont deux réponses dont la connotation positive ou négative était radicalement opposée à celle que nous pensions. Cette question faisait partie d'un ensemble visant à mesurer les aspects négatifs du travail; or ces exemples de réponses montrent que ce que nous avions considéré comme un aspect forcément négatif du travail pouvait être pensé comme un élément positif. Mais une partie des enquêtés ont compris la question comme nous, c'est-à-dire ont compris que répondre «oui» voulait dire qu'on était insatisfait de son travail.

De semblables mésaventures illustrent la difficulté qu'il peut y avoir pour les concepteurs de questionnaire, même les plus avertis, à se débarrasser de l'illusion que des expressions, même courantes, seraient univoques pour toutes les parties de la population.

«La standardisation ostentatoire du protocole et du langage de l'interrogation par questionnaires aveugle plus d'un méthodologue sur les malentendus sémantiques qui s'opèrent au nez et à la barbe de l'interviewer, sous le couvert d'une homogénéité du questionnement, encore plus trompeuse de s'être faite typographique ${ }^{4}$.»

La variété ne s'exprime pas seulement dans la façon de comprendre la question et d'y répondre. Elle peut, de façon plus radicale encore, dénuer la question de sens: nous avons aussi rencontré des enquêtés pour lesquels certaines questions n'étaient tout simplement pas pertinentes.

\section{Questions sans réponse}

«Dans le simple fait de poser la même question à tout le monde, se trouve impliquée l'hypothèse qu'il y a un consensus sur les problèmes, autrement dit, qu'il y a un accord sur les questions qui méritent d'être posées ${ }^{5}$.» Obligé de réfléchir et de donner une réponse à une question qu'il ne se pose pas, voire qu'il ne peut pas se poser, l'enquêté n'offre bien souvent à l'interviewer que son silence.

Ainsi, à la question « $S i$ vous aviez le choix, quelle profession aimeriez-vous exercer?», qui était conçue pour évaluer la distance entre la situation professionnelle actuelle (objectivité) et les aspirations de la personne (subjectivité), les enquêtés de milieux défavorisés ont eu souvent du mal à répondre. Le plus souvent, la personne ouvre de grands yeux, souffle, prend un air étonné, et finit presque toujours par répondre que «de toutes façons, [elle n'avait] pas eu le choix », ce qui doit être considéré comme une réponse, mais qui remet en cause l'idée que le bonheur présent se mesure à ce qu'on espère, puisque certaines personnes n'ont pas éprouvé la possibilité d'espérer autre chose que ce qu'elles sont devenues.

De telles réponses nous montrent qu'on ne peut pas s'extraire de la réalité au même degré selon la classe sociale à laquelle on appartient et que poser des questions sur les rêves ou les espoirs d'une vie autre suppose déjà que la personne peut rêver à une vie différente de la sienne et qu'elle peut le dire à un enquêteur. Or, en dessous d'un certain niveau économique, ces questions n'ont plus de sens: la réalité s'impose avec une telle force (on s'y heurte tous les jours simplement 
pour vivre), qu'on ne peut en faire abstraction et s'autoriser à exprimer ses rêves (s'ils existent) dans une situation aussi officielle qu'une enquête de l'Insee.

On ne peut pourtant pas supprimer totalement de telles questions qui ont leur intérêt pour les catégories de population qui peuvent se les poser, mais il faut laisser s'exprimer la non pertinence d'une question quand elle est avérée. C'est tout l'intérêt des items «sans objet » et «non réponse» qui, malgré les limites qu'ils imposent à l'interprétation des données ${ }^{6}$, sont toujours plus justes qu'une réponse forcée à une fausse question.

Tous ces problèmes posés par les variations qui affectent le sens des mots et la pertinence des problématiques proposées ont déjà été soulignés par la littérature sociologique. Mettant en jeu le langage qui se trouve au cœur même du dispositif de l'enquête par questionnaires, ils sont toujours d'actualité et nous avons observé leur acuité.

Des auteurs comme P. Bourdieu et J.-C. Passeron semblent proposer comme «antidote» (Passeron) un approfondissement de la connaissance préalable du terrain. Certes il est vrai que certaines observations supplémentaires sur le terrain auraient été les bienvenues pour améliorer le questionnaire, mais il ne faut pas se faire d'illusions sur leur utilité: l'intérêt de la démarche statistique est de collecter des données en interrogeant un grand nombre de personnes, ce qu'il est matériellement impossible de faire à partir d'une démarche dite «qualitative».

Il faut prendre en compte au contraire, de façon pragmatique, la présence de l'enquêteur et les conditions matérielles de la situation d'enquête qui sont un aspect fondamental de la passation du questionnaire et qui

6. J.-C. Passeron, op. cit.

7. Gérard Mauger, «Enquêter en milieu populaire», Genèses, n $^{\circ}$ 6, 1991, p. 125-143.

8. G. Mauger, op. cit., p. 129. ont leur rôle à jouer. En effet, construire un questionnaire au plus près du terrain, si tant est que cela soit possible, ne résoudra pas l'ensemble des problèmes de langage dans la 
mesure où le sens des mots se construit dans la situation d'enquête elle-même.

\section{La situation d'enquête}

Comme le souligne Gérard Mauger ${ }^{7}$, la standardisation des données et la division du travail propres aux méthodes quantitatives rendent invisible la situation d'enquête et ont conduit pendant longtemps les chercheurs à la négliger. Quand le chercheur se préoccupe des effets des interactions enquêteur/enquêté, c'est en général dans l'illusion qu'il pourra les neutraliser par toujours plus de standardisation et de division du travail.

Il nous semble au contraire que la seule attitude qui puisse réellement prétendre à la scientificité est de prendre en compte la situation d'enquête en tant que telle: "Les tentatives de neutralisation de l'enquêteur et de la situation d'enquête sont non seulement vouées à l'échec, mais elles véhiculent aussi, le plus souvent en toute méconnaissance, l'illusion qu'existe une "vérité», une «essence» des pratiques, des représentations, des opinions, des enquêtés qu'il faudrait pouvoir observer in situ («entre eux» ou «dans leur for intérieur») et à leur insu. Il s'agit à l'inverse de prendre comme objet d'enquête la situation sociale particulière qu'est la situation d'enquête, les conditions sociales de son établissement, les formes de son déroulement, et de cerner les effets de cette situation particulière sur les "matériaux" recueillis ${ }^{8} . »$

L'exemple de Serge met en évidence l'influence du contexte de la passation sur le recueil des données. La présence de la patronne et de ses enfants autour de la table, le fait que l'enquête se déroule au domicile de l'employeur pendant les heures de travail, ont fait perdre tous ses moyens à Serge, l'enfermant dans le silence face à l'enquêtrice. Il n'y aura aucune trace de cette situa- tion d'enquête particulière dans les données finales de l'Insee alors qu'elle révélait à elle seule tant de choses sur ce qui, précisément, nous intéressait dans cette enquête: le rapport de Serge à son travail. En effet, l'enquêtrice a repris rendez-vous avec lui pour refaire l'enquête dans d'autres conditions (meilleures pour elle).

\section{Enquêter sur l'enquête}

Prendre pour objet d'étude la situation d'enquête en tant que telle, c'est tout d'abord ici prendre en compte notre présence auprès des enquêteurs de l'Insee lors des passations de questionnaire. Nous avons rencontré les enquêteurs par l'intermédiaire des directions régionales de l'Insee en nous présentant comme des étudiants en sociologie désireux de découvrir ce qu'est le travail d'un enquêteur. Par conséquent, c'est le plus souvent une relation de type pédagogique qui s'est instaurée entre eux et nous: les enquêteurs avaient le souci d'expliquer en quoi consistait leur métier et se sont efforcés de se montrer exemplaires sur le terrain. Les observations qui sont présentées ici sont donc celles que nous avons recueillies en les accompagnant chez les enquêtés auxquels nous avons été présentés comme des «stagiaires».

\section{Une enquête officielle}

D'emblée, la passation du questionnaire revêt un caractère officiel: l'enquêteur se présente le plus souvent, afin d'essuyer le moins de refus possible, comme représentant de l'Insee, de l'institution Insee. Avant même le passage de l'enquêteur, chaque enquêté reçoit une lettre-avis qui précise les modalités de l'enquête et souligne son caractère obligatoire, car reconnue d'intérêt général: «Selon la loi $\mathrm{n}^{\circ}$ 51-711 du 7 juin 1951, tout défaut de réponse ou une réponse sciemment inexacte peut entraîner l'application d'une amende administrative». 


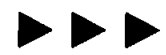

9. Pierre Bourdieu et Gabrielle Balazs, "L'interrogatoire», in P. Bourdieu (dir.), La Misère du monde, Paris, Éd. du Seuil, 1993, pp. 927 et suiv.

10. Extrait d'un compte rendu de passation d'enquête. Nous utilisons ici les comptes rendus rédigés, dans le cadre du séminaire Bonheur et Travail, par tous ceux qui ont eu l'occasion de suivre des enquêteurs. SEGPA: Section d'Enseignement Général et Professionnel Adapté

11. François de Singly, «La gestion sociale des silences», in «Le questionnement et le silence», Consommation, $n^{\circ} 4,1982$.

12. L'intitulé de la première question était: «Qu'est-ce qui, pour vous, est le plus important pour être heureux? » Question ouverte, donc, qui devait être traitée ensuite au moyen de logiciels d'analyse textuelle.
L'enquêteur est donc précédé par la présentation de l'administration Insee. À lui ensuite de jouer avec ce caractère officiel ou non. Par exemple, face au refus d'un homme très âgé (83 ans), une enquêtrice réagit immédiatement en se posant comme représentante de l'institution. Elle précise que l'enquête est obligatoire et emploie presque la menace lorsqu'elle annonce la mise en œuvre d'une procédure complexe et officielle de lettre recommandée si la personne refuse de répondre. La démarche que constitue l'enquête est toujours marquée du sceau de l'officialité. L'aspect institutionnel, même si celui-ci reste implicite, n'est jamais absent et surtout n'est pas sans conséquences sur l'enquête.

L'enquêteur a l'administration de son côté. C'est ce que souligne Bourdieu dans une analyse d'enquêtes administratives sur le RMI': «Si la violence symbolique inhérente à la dissymétrie entre les interlocuteurs, très inégalement pourvus en capital économique et surtout culturel peut s'exercer avec une si parfaite absence de retenue, c'est que les agents chargés de mener l'interrogatoire se sentent mandatés et autorisés par l'État, détenteur du monopole de la violence symbolique légitime, et qu'ils sont en dépit de tout connus et reconnus comme tels.»

Dans certaines conditions, selon l'usage fait par l'enquêteur de son capital de légitimité, selon les capitaux économique et culturel dont dispose l'enquêté pour faire face à une situation officielle et selon les regards que portent l'un sur l'autre l'enquêteur et l'enquêté, la situation d'enquête peut se transformer en véritable «interrogatoire». C'est ce qui se passe lors de l'arrivée chez Mme $\mathrm{H}$.

- L'enquêtrice pénètre donc chez Mme $\mathrm{H}$. avec la fiche du recensement de 1990 , elle demande dès le début de l'entretien si le foyer comporte toujours cinq personnes. Réponse de Mme H.: «Non, la grand-mère est décédée depuis... ». L'entrée en matière, 
entrée en confiance, est d'ores et déjà un échec: nous pénétrons chez $\mathrm{Mme} \mathrm{H}$. en tant que représentantes de l'administration, inspecteurs des vies privées.

Deuxième instant de malaise dès le début de l'entretien: le remplissage du tableau de composition du ménage. L'enquêtrice, se fondant sur la couleur de la peau de Mme H., commence déjà à sortir la carte détaillée des nationalités, manquant ainsi singulièrement de tact puisque celle-ci lui répond «Je suis Réunionnaise, c'est Français ça, madame!»

Dernier moment délicat, qui encore une fois écarte toute mise en confiance initiale: le niveau de diplôme. Mme $\mathrm{H}$. et son mari ont arrêté leurs études avant la fin du primaire mais la même question concernant ses enfants devient embarrassante pour l'enquêtée, qui répond que son fils «a pris du retard», qu'il a été malade et se trouve à présent dans une classe spéciale. L'enquêtrice parvient à déterminer à l'aide des cartes qu'il est en cinquième SEGPA ${ }^{10}$.

Ces premières questions-interrogatoires, d'emblée, jugent Mme $\mathrm{H}$., la réduisent à des cases cochées en référence à la normalité de la société française. Telle qu'elle s'est déroulée pour cette personne, l'entrée en matière de l'enquête (tableau de composition du ménage) a consisté en une catégorisation forcée et violente, négligeant les tentatives de Mme H. pour maintenir son intégrité et ne pas se laisser réduire à des indicateurs mettant en évidence sa position socialement dominée.

Ces «gaffes» de l'enquêteur retentissent sur l'ensemble de la passation du questionnaire: sans un climat de confiance, la personne enquêtée reste sur ses gardes pour protéger ses intérêts matériels et symboliques autant que ses ressources le lui permettent, notamment quand on lui pose des questions ouvertes qui mettent en jeu des facettes très personnelles de son existence (exposer sa conception du bonheur, livrer ses rêves ou ses espoirs...).
La confiance entre enquêté et enquêteur est d'autant plus nécessaire que la relation d'enquête est amenée à ne pas rester privée. F. de Singly compare la situation d'enquête à la relation de confession et note une différence fondamentale: «Dans le confessionnal, une personne ne représente qu'elle-même, dans le sondage, elle est involontairement le "porte-parole" de sa catégorie socio-démographique chargé de répondre pour que le "public" (au sens de ceux qui liront les résultats du sondage) connaisse les attitudes et les comportements de cette catégorie du public $^{11} . »$

La publicité de l'enquête, anticipée par l'enquêté, a des effets en retour sur la situation d'enquête et sur les réponses au questionnaire. Le compte rendu de passation qui suit illustre les modalités concrètes que prend l'adoption d'une ligne de conduite de la part de l'enquêté. Ce qui est le plus frappant lors de ces deux passations c'est la capacité, pour ces deux enquêtées qui disposaient de ressources culturelles suffisantes (il s'agissait d'une pharmacienne et d'une jeune retraitée de la police), à anticiper - en partie - les interprétations qui allaient être faites de leurs réponses et donc de défendre une image d'elles-mêmes positive ou négative. En effet, la première question sur le bonheur ${ }^{12}$ leur fait d'emblée comprendre que le but (ou un des buts) est de voir si elles sont ou non satisfaites de leur travail, de leur vie actuelle, et elles choisissent très vite une stratégie de présentation d'elles-mêmes qui les guide beaucoup plus dans leurs réponses que leurs pratiques réelles.

Cette stratégie de présentation de soi a été amplifiée dans le cas de la pharmacienne, qui a répondu aux questions en présence de son mari. À la question sur le bonheur, elle répond «avoir une vie professionnelle et familiale réussie» et, dès la première question, la ligne de présentation positive sous l'influence du mari est choisie: quand l'enquêtrice parle 


\section{$\rightarrow$}

13. Une des questions demandait à l'enquêté de dire si son travail était ou non une passion pour lui.

14. Extrait d'un compte rendu de passation d'enquête.

15. François de Singly, «Les bons usages de la statistique dans la recherche sociologique ", Economie et Statistique $\mathrm{n}^{\circ} 168,1984$. Voir la journée d'étude Insee-SFS:

«Statistiques et Sociologie», Paris oct. 1982.

16. Erving Goffman, Les Rites d'interaction, Paris, Minuit, 1974.

17. Les enquêteurs sont rémunérés «au questionnaire». de passion ${ }^{13}$, elle sourit, se tourne vers son mari, dit que ça l'était mais ça ne l'est plus. Son mari intervient, dit que ça reste quand même une passion et finalement elle répond «oui». De même, elle présentera la retraite imminente sous un jour positif, et n'évoquera pas les tensions avec son entourage alors qu'il est visible qu'elle appréhende la retraite et que son mari a insisté sur le fait qu'elle est débordée depuis la veille ${ }^{14}$.

La présentation de soi vise donc d'abord à préserver une certaine cohérence personnelle dans l'image de soi. C'est ce que souligne F. de Singly dans son compte rendu de la journée «Statistiques et Sociologie ${ }^{15}$ : toute lecture d'un matériel statistique doit intégrer l'analyse des conditions de son recueil et en particulier prendre en considération le «répertoire figuratif des personnes interrogées », c'est-à-dire d'après la définition de Goffman, «tout ce qu'entreprend une personne pour que ses activités ne fassent perdre la face à personne (y compris elle-même) ${ }^{16}$.»

Nous avons observé ces stratégies de construction de cohérence de soi-même, ce souci de faire "bonne figure », dans des milieux suffisamment dotés culturellement pour maîtriser le sens de l'enquête et de leurs propres réponses. Cette attitude n'est cependant pas possible pour des individus totalement dominés dans la relation d'enquête: les problèmes de compréhension, d'inadéquation du questionnaire à leur expérience de la réalité - problèmes qui interrompent sans cesse le déroulement du questionnaire - sont tels que le maintien d'une ligne de conduite qui suppose une distanciation par rapport au questionnaire n'est pas envisageable. Ainsi, une femme de ménage déclare ne pas travailler le week-end au début du questionnaire; cependant, quand se présente une question sur les activités extra-professionnelles, l'enquêtrice s'aperçoit en faisant le décompte avec l'enquêtée des heures passées à s'occuper de ses 
enfants, qu'elle travaille en fait un week-end sur deux. La domination symbolique que constitue la passation du questionnaire est dans ce cas telle que la construction de la cohérence vis-à-vis de soi-même, même au niveau le plus élémentaire, n'est pas réalisée.

\section{Être enquêteur Insee}

La plupart du temps, notre suivi des passations d'enquête a été l'occasion, pour nous, d'observer en quoi consiste le métier d'enquêteur et, pour les enquêteurs, d'exprimer leur point de vue, de formuler leurs critiques sur l'enquête et, à travers elles, sur les concepteurs de l'enquête.

\section{Le métier d'enquêteur}

Être enquêteur Insee suppose d'acquérir un certain nombre de compétences techniques et relationnelles. Par son tact, ses capacités relationnelles, son savoir-faire, l'enquêteur peut instaurer un climat de confiance dans la situation d'enquête: l'objectif est de «faire passer» le questionnaire. L'importance de la maîtrise de l'enquête par l'enquêteur apparaît a contrario au moment où il y a négligence ou maladresse de sa part comme nous avons eu l'occasion de l'observer parfois.

Par exemple, une enquêtrice fournit à la personne interviewée une carte de réponses erronée (elle aurait da la modifier avant de commencer l'enquête). L'enquêtée choisit une modalité de réponse présente sur la carte mais supprimée dans la version finale de l'enquête. L'enquêtrice, s'apercevant alors de son erreur, présente à la personne le questionnaire lui-même où les réponses sont inscrites dans un certain ordre (or on sait combien l'ordre de présentation des modalités peut influencer la réponse), en petits caractères: la personne interviewée cherche ses lunettes, doit modifier sa réponse sans trouver de terme qui la satisfasse autant que le premier.
Autant d'interruptions, de lenteurs dans l'enquête et de lassitude chez l'enquêtée.

Ainsi, le métier d'enquêteur exige une vigilance constante: la moindre négligence matérielle se répercute sur le déroulement du questionnaire et influence la relation d'enquête. En outre les questionnaires de l'Insee supposent souvent que les enquêteurs sachent manipuler des outils statistiques spécifiques, parfois complexes: la procédure de tirage Kish surtout tirage au sort de la personne interviewée dans le ménage pour assurer une représentativité nationale de l'échantillon, c'est-à-dire pour éviter que les femmes au foyer ou les retraités, plus disponibles, ne soient sur-représentés suscite un certain nombre de contraintes et problèmes techniques. Certains enquêteurs avaient des difficultés à définir qui était éligible pour le questionnaire (parce que les nomenclatures laissent toujours des marges d'indétermination). Parfois, la solution de facilité consistait à choisir la personne qui était disponible pour répondre.

En effet, la procédure Kish est très contraignante pour l'enquêteur qui doit parfois se déplacer deux fois dans le même ménage et négocier un second passage auprès des enquêtés. Pour encourager les enquêteurs à la respecter, l'Insee a tenté de mettre en place un système de primes: chaque enquêteur, s'il revenait dans un même ménage, était payé $60 \mathrm{~F}$ de plus que l'enquête simple (rémunérée, elle, $120 \mathrm{~F}$ ). Mais, ce système de primes n'étant, en réalité, pas contrôlable (on ne peut pas vérifier que l'enquêteur est bien allé deux fois dans le même ménage), il a été supprimé. On peut donc comprendre que, pris dans des contraintes de temps et d'argent ${ }^{17}$, les enquêteurs en viennent à la solution de facilité évoquée plus haut.

Cet exemple met en évidence combien les enquêteurs sont pris dans un faisceau de contraintes et d'exigences contradictoires: les responsables de l'Insee et les concepteurs des 


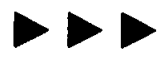

18. Lena Tenevic et Florence Weber, «La délégation du travail de terrain en sociologie qualitative», Genèses, n8, 1992, pp. 132-145.

19. Extrait d'un compte rendu de passation d'enquête.

20. Il s'agit d'un questionnaire sur lequel sont portés, à côté des questions concernées (dans une bulle du type de celles qu'on trouve dans les bandes dessinées), les commentaires ou les explications destinées aux enquêteurs. enquêtes, prisonniers de l'illusion de la neutralité de la situation d'enquête, voudraient qu'ils se comportent comme de simples courroies de transmission entre concepteurs et enquêtés alors que la qualité dans le recueil des données suppose justement de la part des enquêteurs une réflexion sociologique sur leur pratique et sur leur rôle dans la situation d'enquête.

Lena Tenevic, dans un entretien avec F. Weber, souligne le décalage entre sa conscience professionnelle (sa conception du travail bien fait) et sa place objective dans le dispositif d'enquête: «Un jour, j'ai parlé de mon travail en réunion syndicale, un camarade m'a répondu: "Tu en fais trois fois trop; tu n'as pas à rentrer dans la problématique des chercheurs lorsque tu fais des enquêtes". Il avait raison. Mais moi, pour mettre en œuvre mes compétences, j'étais obligée de me mettre dans la peau... de dire c'est ma recherche, alors que je savais très bien que c'était pas la mienne. Là, effectivement, tu te fais avoir ${ }^{18}$.»

Quelles relations avec les concepteurs de l'enquête?

Les enquêteurs que nous avons rencontrés savaient que nous avions plus ou moins participé à la conception du questionnaire en tant qu'étudiants: nous étions donc en lien avec les concepteurs de l'enquête, sans toutefois porter l'entière responsabilité de la lettre du questionnaire. Ils nous ont donc considérés comme d'éventuels médiateurs vers les «chercheurs parisiens » et nous ont livré leur point de vue sur les concepteurs.

Deux attitudes principales ont été relevées. La première consiste en un point de vue très critique: l'enquête est jugée trop loin de la réalité. Dans cette perspective, une jeune enquêtrice fort mécontente profite de la présence d'un étudiant pour dire aux concepteurs ce qui ne convient pas: le questionnaire 
est mal formulé, dans un vocabulaire d'énarques, d'intellectuels parisiens... On retrouve ainsi chez un certain nombre d'enquêteurs une certaine mythologie du terrain - il s'agit «d'être dans la vie » - qui accentue la distance entre enquêteurs sur le terrain et concepteurs loin de la réalité. Les enquêteurs critiquent alors la difficulté de circulation de l'information dans la hiérarchie Insee du bas vers le haut: les concepteurs ne prennent pas assez en considération les remarques provenant des enquêteurs.

Mais d'autres enquêteurs adoptent la démarche inverse; ils tendent à nier la distance entre eux et les concepteurs. - Elle (l'enquêtrice) ne semble pas du tout gênée par ma présence: elle a plutôt tendance, tout en insistant sur sa pratique de terrain, à se présenter comme une «intellectuelle», capable de réfléchir sur sa pratique. Elle déplore surtout l'isolement des enquêteurs et le peu d'informations dont ils disposent sur les objectifs de l'enquête. Sa stratégie de valorisation ne repose donc pas sur une opposition entre enquêteurs, concepteurs et direction de l'Insee mais au contraire sur une tentative d'intégration ${ }^{19}$... Sa critique porte alors moins sur le manque de circulation des informations du bas vers le haut mais bien plus du haut vers le bas.

Ces deux attitudes renvoient à un manque de coopération entre enquêteurs et concepteurs de l'enquête. D'un côté comme de l'autre, ce manque a été ressenti: nous nous sommes aperçus lors du dépouillement que le recueil des données aurait gagné à une meilleure présentation de la problématique du questionnaire auprès des enquêteurs. Ainsi, la plupart des enquêteurs n'ont pas saisi toute l'importance que nous accordions au recueil intégral des réponses aux questions ouvertes. Par exemple, à la question «Si vous aviez le choix, quelle profession aimeriezvous exercer?», un enquêté répond: «le même métier, je l'ai choisi, il me plaît», l'enquêteur, lors de la retranscription, synthétise: «le même métier, il me plaît». Il supprime ainsi une précision fondamentale pour nous: la notion de choix qui s'inscrivait dans la problématique du rapport entre bonheur et travail. Il est certain qu'une telle attitude est également liée aux contraintes fortes (notamment la contrainte temporelle) qui pèsent sur les enquêteurs, mais une meilleure communication entre concepteurs et enquêteurs ne pourrait qu'améliorer la qualité des données.

En l'état actuel des choses, la coopération entre enquêteurs et concepteurs est en effet entravée par le problème des formations dispensées aux enquêteurs : celles-ci sont de plus en plus brèves (deux heures dans chaque direction régionale) ou remplacées par des formations en kit, du type questionnaire à bulles ${ }^{20}$. De plus, la perte d'information est énorme à cause de l'organisation en cascade, du concepteur vers des représentants des Directions Régionales de l'Insee puis de ces représentants jusqu'aux enquêteurs. Or, la formation est justement le moment où l'ensemble des participants au processus d'enquête devraient se rencontrer et coordonner leur action. Sans que cela résolve tous les problèmes, on peut reprendre la proposition du responsable de l'animation de la collecte à l'Insee: associer dès le début de la conception du questionnaire un représentant des enquêteurs, celuici pouvant alors jouer un rôle de relais entre enquêteurs et concepteurs.

À l'issue des passations de notre questionnaire, les difficultés rencontrées nous montrent qu'une approche qualitative, même plus poussée que la nôtre (pourtant guère fréquente pour ce genre d'enquête), ne nous aurait pas permis d'appréhender correctement les liens entre travail et bonheur pour 
tous les milieux sociaux. Sur un échantillon de 8000 ménages, il devait forcément y avoir des enquêtés pour lesquels les questions que nous posions n'étaient pas en adéquation avec leur expérience de la réalité, voire totalement décalées par rapport à leur horizon de vie.

«L'enquête sur l'enquête», lorsque nous avons suivi les enquêteurs dans leur travail, confirme qu'il est nécessaire de prendre en compte les particularités des conditions du recueil de l'information dans une enquête par questionnaire. Face à un fichier statistique de près de 6000 réponses, il faut toujours garder en mémoire que ce fichier est le résultat d'une multitude de relations sociales, de compromis, de distorsions, de malentendus qui se sont noués entre les enquêtés, les enquêteurs, l'Insee en tant qu'administration et les concepteurs du questionnaire, même le plus standardisé.

Il ne s'agit pas de corriger les insuffisances du questionnaire par une approche qualitative illusoirement exhaustive mais de souligner les limites de l'une et de l'autre méthode. Quelle que soit l'approche utilisée (qualitative ou quantitative), nos observations confirment que toute situation d'enquête, comme toute situation sociale, n'est jamais neutre: "La vérité" des enquêtés ne gît pas plus dans les pratiques qui ont cours ou dans les propos qui sont tenus hors de la présence de l'enquêteur que dans la situation d'enquête ${ }^{21}$ ».

Il n'est de sociologie que des enquêtés. Cependant, la prise en compte de la totalité de la situation d'enquête suppose de sortir de l'ombre le point aveugle inhérent à toute connaissance sociologique: l'enquêteur. Dans cette perspective, ces quelques réflexions mériteraient d'être prolongées par d'autres observations (du type "enquête sur l'enquête») visant à constituer une véritable sociologie des enquêteurs. 